\title{
ALDH1B1 predicts poor survival for locally advanced nasopharyngeal carcinoma patients
}

\author{
Tao Zhu ${ }^{1 \#}$, Jun-Yan He ${ }^{1 \#}$, Yan-Ping Liu ${ }^{2}$, Kun Deng ${ }^{3}$, Jian-Hong Zuo ${ }^{3}$, Xiao-Hong Ai $^{1 \wedge}$ \\ ${ }^{1}$ Department of Radiation Oncology, The First Affiliated Hospital, Hengyang Medical School, University of South China, Hengyang, China; \\ ${ }^{2}$ Department of Nosocomial Infection Control, The First Affiliated Hospital, Hengyang Medical School, University of South China, Hengyang, \\ China; ${ }^{3}$ The Laboratory of Translational Medicine, Hengyang Medical School, University of South China, Hengyang, China \\ Contributions: (I) Conception and design: JH Zuo, XH Ai; (II) Administrative support: JH Zuo, XH Ai; (III) Provision of study materials or patients: \\ XH Ai; (IV) Collection and assembly of data: T Zhu, JY He; (V) Data analysis and interpretation: T Zhu, JY He, YP Liu, K Deng; (VI) Manuscript \\ writing: All authors; (VII) Final approval of manuscript: All authors. \\ \#These authors contributed equally to this work. \\ Correspondence to: Xiao-Hong Ai. Department of Radiation Oncology, The First Affiliated Hospital, Hengyang Medical School, University of South \\ China, Hengyang 421001, China. Email: aixiaohong113@163.com; Jian-Hong Zuo. The Laboratory of Translational Medicine, Hengyang Medical \\ School, University of South China, Hengyang 421001, China. Email: 632138414@qq.com.
}

Background: Distant metastasis and local recurrence remain the major reasons of treatment failure in locally advanced nasopharyngeal carcinoma (NPC). Therefore, exploring novel biomarkers for prognosis and sensitivity of radiotherapy in locally advanced NPC is crucial. This retrospective study evaluates the expression and prognostic value of aldehyde dehydrogenase 1B1 (ALDH1B1) for locally advanced NPC patients.

Methods: Sixty-seven locally advanced NPC patients and 22 chronic nasopharyngitis patients between September 2012 to November 2016 at The First Affiliated Hospital of University of South China were enrolled in this study. The expression of ALDH1B1 in tumor tissues were detected by using immunohistochemistry (IHC).

Results: Significant difference was observed between NPC groups and Pharyngitis tissues groups, and NPC groups has a higher ratio of high ALDH1B1 expression. ALDH1B1 expression were significantly associated with age and radiotherapy response. The Kaplan-Meier analysis indicated that patients with high ALDH1B1 expression had a poor prognosis both in overall survival (OS) and progression-free survival (PFS). Univariate analysis found that age, radiotherapy response and ALDH1B1 expression were correlated with OS. Besides, factors affecting PFS are radiotherapy response and ALDH1B1 expression. Multivariate analysis revealed that radiotherapy response and ALDH1B1 expression were the independent prognostic factors for OS, whereas radiotherapy response was for PFS.

Conclusions: The expression of ALDH1B1 was correlated with age and radiotherapy response. Patients with high ALDH1B1 expression show a poor prognosis both in OS and DFS. ALDH1B1 expression were the independent prognostic factors for OS.

Keywords: Aldehyde dehydrogenase 1B1 (ALDH1B1); nasopharyngeal carcinoma (NPC); prognosis; radioresistance

Submitted Sep 16, 2021. Accepted for publication Dec 26, 2021.

doi: $10.21037 /$ tcr-21-1979

View this article at: https://dx.doi.org/10.21037/tcr-21-1979

^ ORCID: Jun-Yan He, 0000-0002-8334-3484; Xiao-Hong Ai, 0000-0002-6217-1935. 


\section{Introduction}

In 2018 , about 12,900 patients were diagnosed as nasopharyngeal carcinoma (NPC), which represent less than one percent of all new-onset cancers (1). However, its geographical global distribution is extremely unbalanced. Over $70 \%$ of new cases occur in south China and some regions of southeastern Asia. Despite the incidence and mortality had declined over the past decade, NPC still largely threaten local people's health (2). For the locally advanced NPC patients, current treatment strategies are radiotherapy and chemotherapy (induction, concurrent and adjuvant) (3). In addition, immunotherapy has achieved breakthroughs in anticancer treatment and has been considered as a promising future direction in the treatment of NPC (4). However, distant metastasis and local recurrence remain the major reasons of treatment failure in locally advanced NPC (5). Therefore, exploring novel biomarkers for prognosis and sensitivity of radiotherapy in locally advanced NPC is crucial.

Considerable evidences have supported that tumorigenesis and therapy resistance were associated with cancer stem cells (CSC) (6-8). The activity of CSC marker aldehyde dehydrogenase (ALDH), especially its isoform 1 (ALDH1), have been proven to be consistent with CSC activity in a variety of cancers. The ALDH1 plays a critical role in metabolizing and detoxifying the endogenous and exogenous aldehydes. The expression level of ALDH1 was also significantly associated with patients' prognosis in various cancers (9-11). Recent studies have indicated that ALDH1B1, a member of ALDH1 family, was overexpressed in various cancers. Meanwhile it plays an important role in tumorigenesis and therapy resistance (12-14). In addition, the high expression of ALDH1B1 was also associated with unfavored prognosis $(15,16)$. However, the expression of ALDH1B1 and its prognostic role in NPC are still unclear.

In the present study, we have investigated the expression of ALDH1B1 and its prognostic role in locally advanced NPC. We found that ALDH1B1 was up-regulated in locally advanced NPC and associated with radiotherapy response. Patients with high ALDH1B1 expression had a poor survival. The results may provide novel prognostic marker and a potential therapeutic target for locally advanced NPC. We present the following article in accordance with the REMARK reporting checklist (available at https://tcr. amegroups.com/article/view/10.21037/tcr-21-1979/rc).

\section{Methods}

\section{Patients}

We retrospectively collected 67 locally advanced NPC patients and 22 chronic nasopharyngitis patients between September 2012 to November 2016 at The First Affiliated Hospital of University of South China. The eligibility criteria were as follows: (I) all patients with pathologically confirmed by pathology examination and the paraffin-embedded tissue samples were available for immunohistochemistry (IHC); (II) no history of other cancers; (III) no evidence of distant metastases; (IV) locally advanced disease (stage III or IVa) diagnosed by magnetic resonance imaging (MRI); (V) all patients received radical concurrent chemoradiotherapy in our hospital. We used the $8^{\text {th }}$ American Joint Committee on Cancer (AJCC) staging system to classify the TNM stage of all the locally advanced NPC patients. This study was conducted in accordance with the Declaration of Helsinki (as revised in 2013) and was approved by the Ethics Committee of The First Affiliated Hospital of University of South China (No. 2020110512007). The requirement for informed consent was waived.

\section{Treatments}

All patients had received external beam intensity-modulated radiation therapy (IMRT) concurrently combined with platinum-based chemotherapy. Radiotherapy was applied as follows: planned targeted volume (PTV)nx received 66-72 Gy, PTVnd received 64-70 Gy, PTV1 received 60-64 Gy, PTV2 received 50-54 Gy. The dosage of each PTV were delivered in 30 or 31 fractions over 6 weeks. During the period of radiotherapy, concurrent platinumbased chemotherapy (mainly DDP or DP) was also delivered every week. Besides, some patients (10 of 67) received two periods of induction chemotherapy.

We used response evaluation criteria in solid tumors (RECIST 1.1) to evaluate the efficacy of treatments (17). All patients were divided into radiosensitive and radioresistance groups according to the therapeutic effects after the completion of concurrent chemoradiotherapy. Radiosensitive patients were defined as patients with complete response or partial response over 12 months after the end of radical concurrent chemoradiotherapy. Radioresistance patients were defined as patients with progressive response or stable disease within 12 months after the end of radical concurrent 
chemoradiotherapy $(18,19)$.

\section{IHC}

IHC was performed according to the standard procedures. Briefly, all the paraffin-embedded specimen blocks were sliced into $4-\mu \mathrm{m}$-thick sections. Xylene was used to deparaffinize these sections and rehydrate them by alcohol gradient. Then, the paraffin sections were rinsed in $0.05 \%$ phosphate-buffered saline (PBS) and blocked with hydrogen peroxidase at room temperature for endogenous peroxidase ablation. Before IHC, heat retrieval with sodium citrate buffer and ethylenediaminetetraacetic acid (EDTA) repair were carried out. The samples were incubated with the anti-ALDH1B1 antibody (22220-1-AP, 1:300; Proteintech, China) overnight at $4{ }^{\circ} \mathrm{C}(16-18 \mathrm{~h})$. After washing, the samples were incubated with the secondary antibody for $20 \mathrm{~min}$, followed by color development with 3,3-diaminobenzidine (DAB) at room temperature in darkness for $3 \mathrm{~min}$. The sections were counterstained with hematoxylin.

Two experienced pathologists who were blinded to the clinical data, independently evaluated the expression of ALDH1B1. The percentage of ALDH1B1 positive cells was graded as follow: $0=<5 \%, 1=5-24 \%, 2=25-49 \%, 3=50-74 \%$, and $4=75-100 \%$. The intensity of ALDH1B1 staining was graded as follow: $0=$ no staining, $1=$ low intensity, and $2=$ high intensity. The result was determined by the sum of these two scores, and this sum is referred to as the combined IHC staining score (CSS) (20). CSS was applied to divide the ALDH1B1 expression into two groups (low expression and high expression), low ALDH1B1 expression is from 0 to 3 while high ALDH1B1 expression is from 4 to 7 .

\section{Follow-up}

After radical concurrent chemoradiotherapy, patients were followed-up every 3 months in the first year, every 6 months for the next 2 years, and finally at 1 -year intervals until death. Radiologically examination was performed routinely during each follow-up visit. The primary endpoints of this study were overall survival (OS) and progression-free survival (PFS). The OS was defined as the time between the completion of radical concurrent chemoradiotherapy and death from any cause, the PFS was defined as the time between the completion of radical concurrent chemoradiotherapy and the date of progression or death.

\section{Statistical analysis}

Categorical variables were compared by $\chi^{2}$ test or the Fisher's exact test. Kaplan-Meier curves were used to construct the survival curves and log-rank tests were used to analyze the difference. The Cox proportional hazards model was used for univariate and multivariate analysis. All the tests of significance were two-tailed: differences at $\mathrm{P}$ values $<0.05$ were considered significant. SPSS (version 22.0) was used to perform the statistical analyses.

\section{The Cancer Genome Atlas (TCGA) data analysis}

The Gene Expression Profiling Interactive Analysis (GEPIA), a user-friendly data portal, were used to analyze the mRNA expression of ALDH1B1 and its prognostic value in head and neck squamous cell carcinoma (HNSC) from the database of TCGA (21). GEPIA is available at http://gepia.cancer-pku.cn/.

\section{Results}

\section{Patient characteristics}

From September 2012 to November 2016, 67 pathologically confirmed locally advanced NPC patients (48 males and 19 females) who received radical concurrent chemoradiotherapy were enrolled in the study. The patients' characteristics are summarized in Table 1 . The median follow-up time was 55.6 (range, 9.7 to 96.7) months. The median age was 50 (range, 24 to 74 ) years. Thirty patients $(44.8 \%)$ had a smoking history. According to the World Health Organization (WHO), the histologic subtypes of NPC were classified as keratinizing squamous cell carcinoma (WHO type I), nonkeratinizing differentiated carcinoma (WHO type II), and nonkeratinizing undifferentiated carcinoma (WHO type III). Of all the patients, 26 patients were the WHO II and 41 patients were the WHO III. The clinical TNM stages III and IVa were 47 (70.1\%) and $20(29.9 \%)$, respectively. With respect to the radiotherapy response, the radiosensitive and radioresistance patients were $45(67.2 \%)$ and $22(32.8 \%)$, respectively.

\section{Expression of ALDH1B1 in NPC}

According to the results of IHC staining, ALDH1B1 protein was mainly located in cell cytosol (Figure 1). High ALDH1B1 expression was detected in $43.3 \%(29 / 67)$ NPC patients and $18.2 \%(4 / 22)$ chronic nasopharyngitis patients, which 
Table 1 Patients' characteristics

\begin{tabular}{|c|c|}
\hline Characteristics & N (\%) \\
\hline \multicolumn{2}{|l|}{ Age (years) } \\
\hline$\leq 50$ & $34(50.7)$ \\
\hline$>50$ & $33(49.3)$ \\
\hline \multicolumn{2}{|l|}{ Sex } \\
\hline Male & $48(71.6)$ \\
\hline Female & $19(28.4)$ \\
\hline \multicolumn{2}{|l|}{ Smoking } \\
\hline Yes & $30(44.8)$ \\
\hline No & $37(55.2)$ \\
\hline \multicolumn{2}{|c|}{ WHO pathologic classification } \\
\hline WHO II & $26(38.8)$ \\
\hline WHO III & $41(61.2)$ \\
\hline \multicolumn{2}{|l|}{ T stage } \\
\hline T1-T2 & $29(43.3)$ \\
\hline T3-T4 & $38(56.7)$ \\
\hline \multicolumn{2}{|l|}{$\mathrm{N}$ stage } \\
\hline No-N2 & $54(80.6)$ \\
\hline N3 & $13(19.4)$ \\
\hline \multicolumn{2}{|l|}{ TNM stage } \\
\hline III & $47(70.1)$ \\
\hline IVa & $20(29.9)$ \\
\hline \multicolumn{2}{|l|}{ Radiotherapy } \\
\hline Sensitive & $45(67.2)$ \\
\hline Resistant & $22(32.8)$ \\
\hline \multicolumn{2}{|c|}{ ALDH1B1 expression } \\
\hline Low & $38(56.7)$ \\
\hline High & $29(43.3)$ \\
\hline
\end{tabular}

WHO, World Health Organization; ALDH1B1, aldehyde dehydrogenase $1 \mathrm{~B} 1$.

had significant difference (Figure $2 A$ ). We also investigate the expression of ALDH1B1 in HNSC from the database of TCGA. The results showed that ALDH1B1 was upregulated in HNSC compared with normal tissue (Figure 2B).

The clinical characteristics between the high ALDH1B1 expression and low ALDH1B1 expression groups were also compared, and the results are listed in Table 2. ALDH1B1 expression was significantly correlated to age $(\mathrm{P}<0.001)$ and radiotherapy response $(\mathrm{P}=0.034)$. No significant differences were found in sex, history of smoking, pathological type, and TNM stage.

\section{Prognostic value of ALDH1B1 expression}

The 5-year PFS for low ALDH1B1 expression group and high ALDH1B1 expression group was $68.3 \%$ and $44.3 \%$, respectively. The 5-year OS for low ALDH1B1 expression group and high ALDH1B1 expression group was 86.8\% and $45.9 \%$, respectively. The Kaplan-Meier survival curves showed that patients with high ALDH1B1 expression had an unfavorable prognosis both in PFS and $\mathrm{OS}(\mathrm{P}=0.017$ and $\mathrm{P}<0.001$, respectively) (Figure $3 A, 3 B$ ). We also investigate the prognostic value of ALDH1B1 in HNSC from the date base of TCGA. The results indicated that no statistic difference was found between high and low ALDB1B1 expression group in DFS (Figure 3C). However, patients with high ALDH1B1 expression had a worse prognosis in OS $(\mathrm{P}=0.0034)$, which was in accordance with our results (Figure 3D).

To further investigate the prognostic value of ALDH1B1 expression, Cox proportional hazards model were performed to analyze the prognostic factors that affect the PFS and OS (Tables 3,4). The factors influencing patients' survival were evaluated by univariate analysis. As listed in Table 3, factors correlated with PFS are radiotherapy response $(\mathrm{P}<0.001)$ and ALDH1B1 expression $(\mathrm{P}=0.021)$. Our results showed that age $(\mathrm{P}=0.022)$, radiotherapy response $(\mathrm{P}=0.001)$ and ALDH1B1 expression $(\mathrm{P}=0.002)$ were significantly correlated with patients' OS (Table 4). The multivariate analysis, which consisted of all the positive prognostic factors in univariate analysis, revealed that radiotherapy response $(\mathrm{P}<0.001)$ was the independent prognostic factors for PFS, whereas radiotherapy response $(\mathrm{P}=0.004)$ and $\mathrm{ALDH} 1 \mathrm{~B} 1$ expression $(\mathrm{P}=0.036)$ were the independent prognostic factors for OS (Tables 3,4).

\section{Discussion}

NPC is the one of the most common malignancy in otolaryngology head and neck, and over $70 \%$ of new NPC occurred in south China and some regions of southeastern Asia (22). As NPC's location is difficult to detect and early symptoms is no obvious, NPC patients were usually diagnosed at an advanced stage. Radiotherapy are the primary therapeutic option for locally advanced 
A
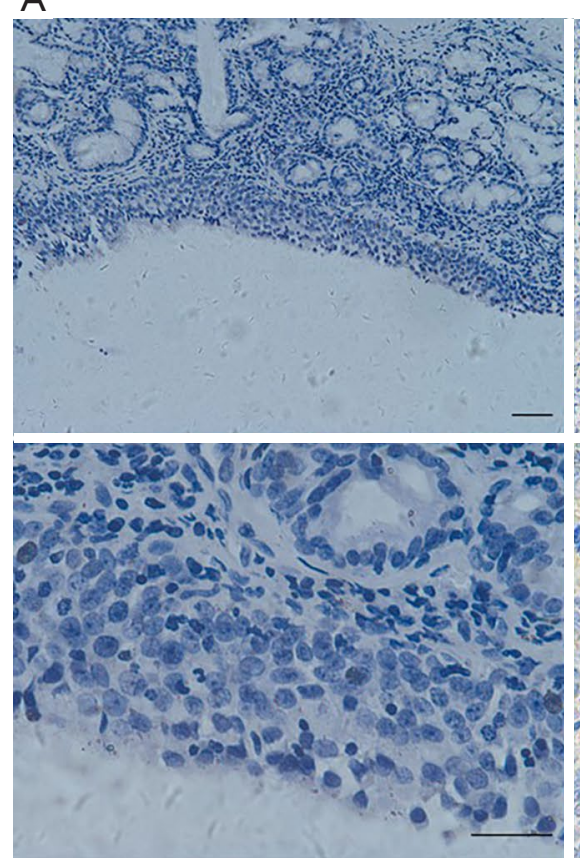

B

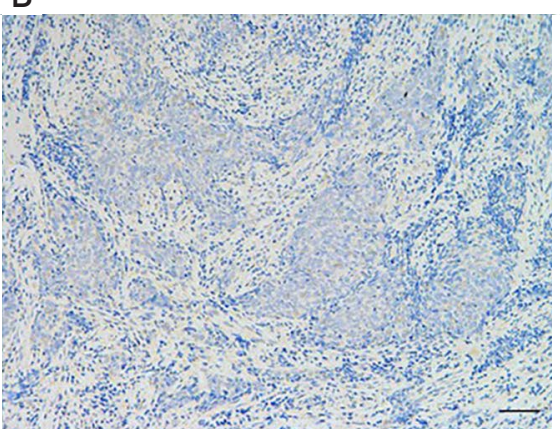

C

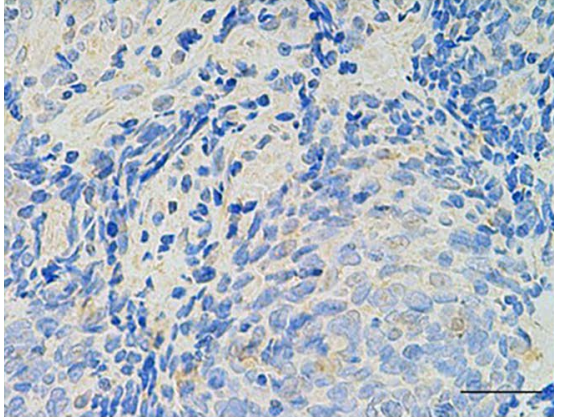

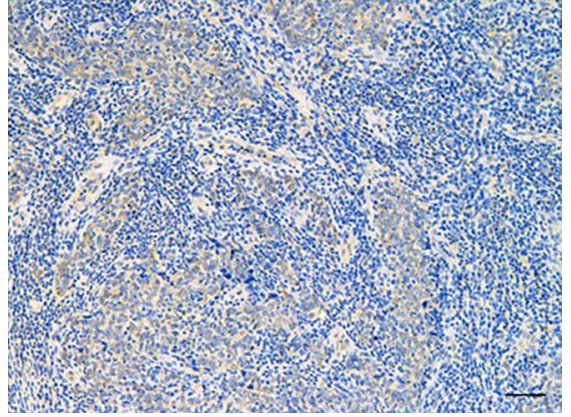

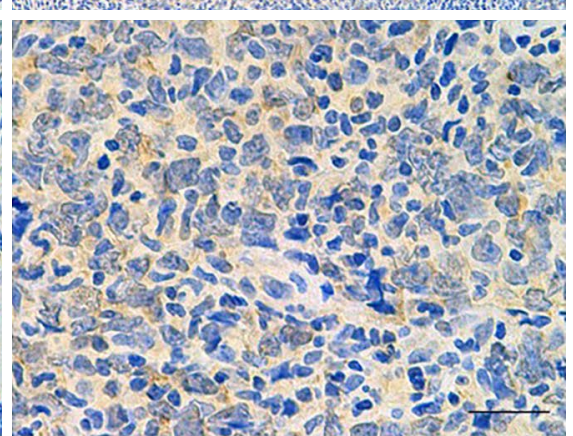

Figure 1 IHC staining of ALDH1B1 expressions in locally advanced NPC and chronic nasopharyngitis tissues (upper: magnification 100x, lower: magnification 400x). (A) ALDH1B1 expression in chronic nasopharyngitis tissues. (B) Low ALDH1B1 expression in locally advanced NPC tissues. (C) High ALDH1B1 expression in locally advanced NPC tissues. IHC, immunohistochemistry; ALDH1B1, aldehyde dehydrogenase 1B1; NPC, nasopharyngeal carcinoma.

A

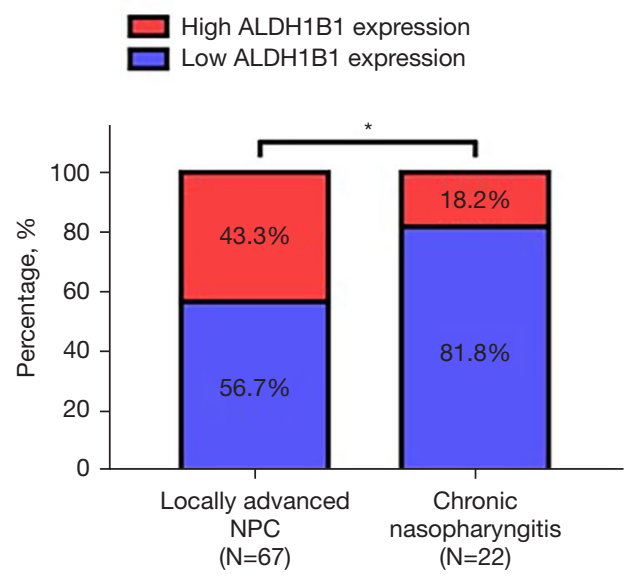

B

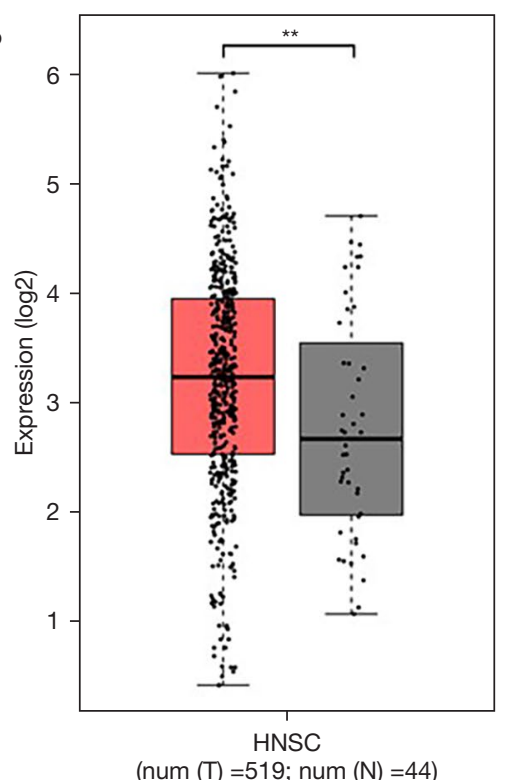

Figure 2 The expression level of ALDH1B1 in different tissues. (A) ALDH1B1 was significantly up-regulated in locally advanced NPC, compared with chronic nasopharyngitis tissues. (B) ALDH1B1 was significantly up-regulated in HNSC, compared with normal tissues. ${ }^{*} \mathrm{P}<0.05 ;{ }^{* *} \mathrm{P}<0.01$. ALDH1B1, aldehyde dehydrogenase 1B1; NPC, nasopharyngeal carcinoma; HNSC, head and neck squamous cell carcinoma. 
Table 2 Correlation between the clinicopathologic features and expression of ALDH1B1

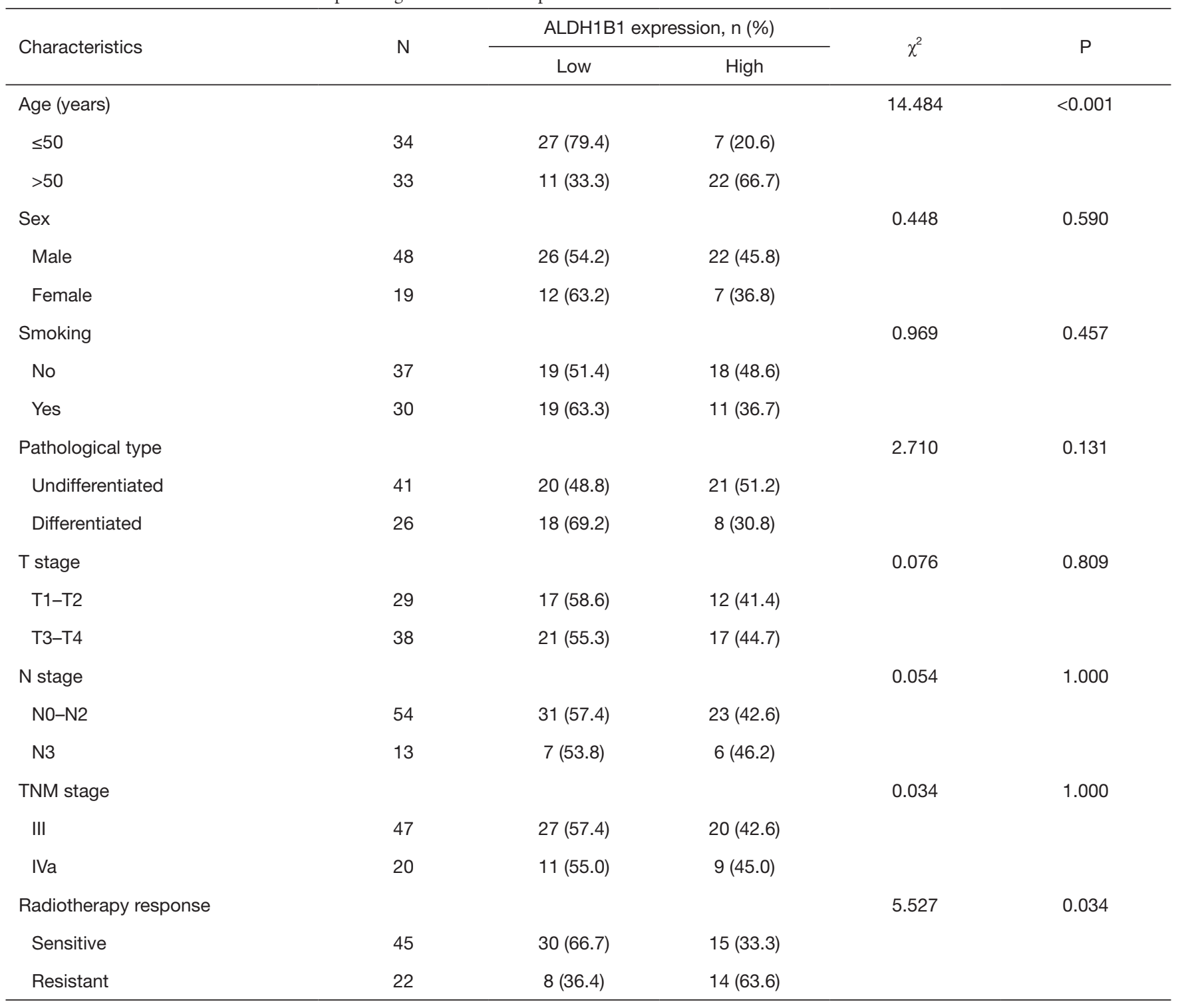

ALDH1B1, aldehyde dehydrogenase 1B1.

NPC (3). Unfortunately, some NPC patients are resistant to radiotherapy, which leads to the treatment failure and increase the risk of distant metastasis or local recurrence (5). Therefore, it is of great significance to explore novel biomarkers to predict the prognosis and radiotherapy response of NPC patients. It also provides individual treatment strategy for the NPC patients who cannot benefit from radiotherapy.

The main reasons for the treatment failure of locally advanced NPC were resistance to radiotherapy, local recurrence, distant metastasis and avoidance of immunological surveillance (22). All these failures could be attributed to the CSC. CSC has strong self-renewal and differentiation potential. Moreover, it can regulate various cellular signaling pathways (such as $\mathrm{Wnt} / \beta$-catenin, $\mathrm{PI} 3 \mathrm{~K} / \mathrm{AKT} / \mathrm{mTOR}$, Hedgehog, etc.) to increase the local recurrence, distant metastasis and therapy resistance of tumor (23). ALDH, a marker in many CSC, played a critical role in drug and radiation resistance. Accumulating evidences have suggested that the failure of cancer treatment such as therapy resistance or disease progression could be contributed to the activity of ALDH (24-26). 

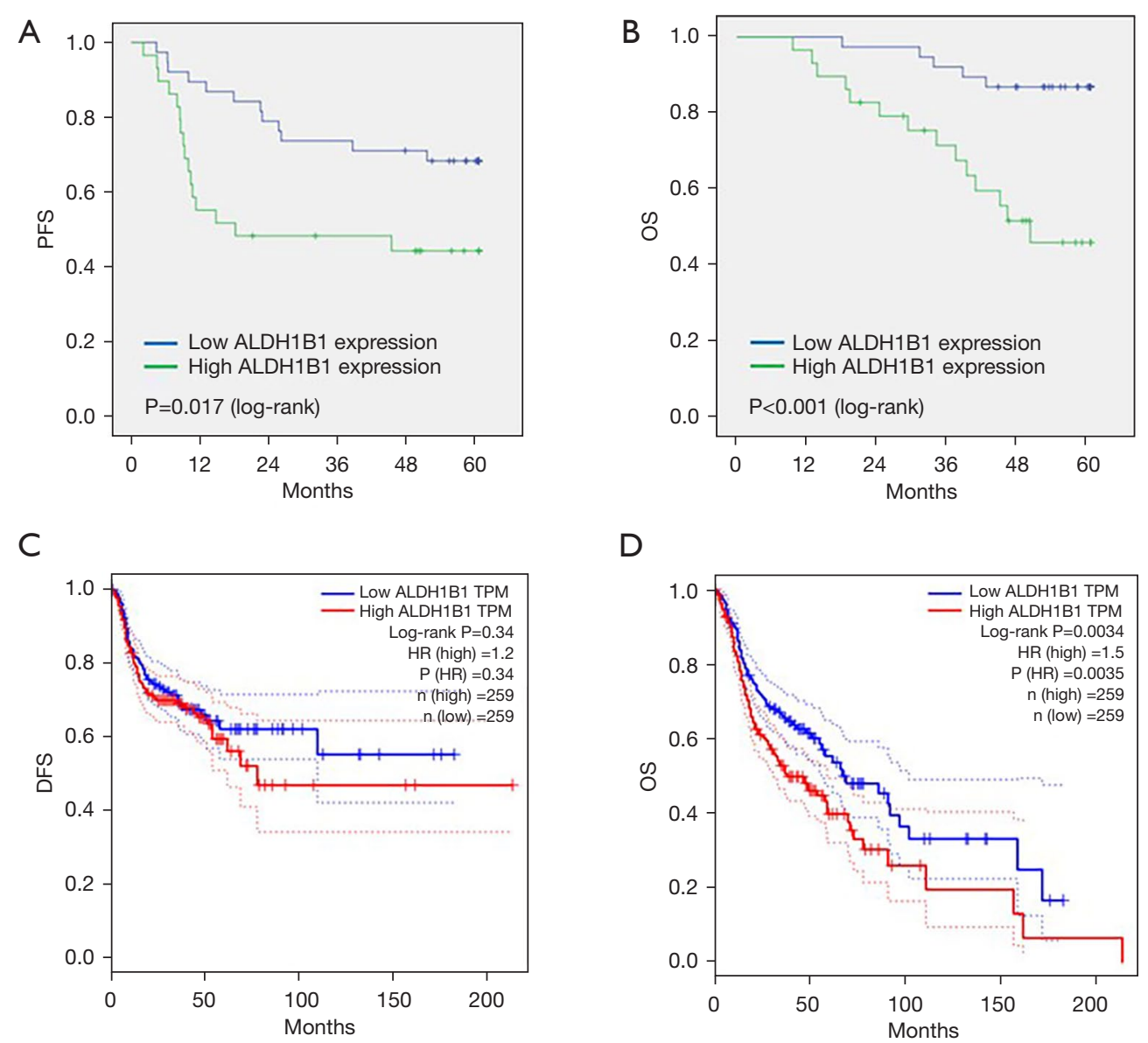

Figure 3 Correlation of ALDH1B1 and prognosis. (A,B) Locally advanced NPC patients with high ALDH1B1 expression had an unfavorable prognosis both in PFS and OS ( $\mathrm{P}=0.017$ and $\mathrm{P}<0.001$, respectively). (C) No significant difference was found between ALDH1B1 expression and DFS in HNSC patients. (D) HNSC patients with high ALDH1B1 expression had a worse prognosis in OS (P=0.0034). ALDH1B1, aldehyde dehydrogenase 1B1; NPC, nasopharyngeal carcinoma; PFS, progression-free survival; OS, overall survival; DFS, disease-free survival; HNSC, head and neck squamous cell carcinoma.

Previous studies revealed that ALDH can eliminate the oxidative stress and increase the drug resistance (25). Some studies indicated that ALDH can enhance resistance to radiation through removing the free radicals generated by the radiolysis of water (26).

ALDH1B1 is a member of ALDH superfamily. Many studies have shown that ALDH1B1 was tightly associated with tumorigenesis and therapy resistance $(12,13,27)$. It has been reported that up-regulating the expression of ALDH1B1 could enhance the resistance of colorectal cancer cells against doxorubicin, fluorouracil and etoposide (12). Singh et al. demonstrated that ALDH1B1 was highly expressed in human colonic adenocarcinomas and could promote colon cancer tumorigenesis by modulating the
Wnt/ $\beta$-catenin, Notch and PI3K/Akt signaling pathways (13). Wang et al. found that ALDH1B1 promote the tumorigenesis of osteosarcoma and was significantly correlated with poor prognosis in osteosarcoma patients (27). However, the expression of ALDH1B1 and its prognostic role in NPC are still unclear.

In this study, we reported the first study focusing on the impact of ALDH1B1 expression in prognosis and the association with clinical characteristics for locally advanced NPC. The expression level of ALDH1B1 in 67 locally advanced NPC and 22 chronic nasopharyngitis tissues were evaluated by IHC. Our results showed that ALDB1B1 was significantly up-regulated in NPC tissues compared with pharyngitis tissues, which indicated that ALDH1B1 may 
Table 3 Cox regression for PFS analysis

\begin{tabular}{|c|c|c|c|c|c|c|}
\hline Characteristics & \multicolumn{3}{|c|}{ Univariate } & \multicolumn{3}{|c|}{ Multivariate } \\
\hline Age (years) & 2.010 & $0.940-4.299$ & 0.072 & & & \\
\hline Sex & 2.033 & $0.773-5.350$ & 0.151 & & & \\
\hline Smoking & 0.753 & $0.353-1.609$ & 0.464 & & & \\
\hline T stage & 1.513 & $0.697-3.285$ & 0.295 & & & \\
\hline $\mathrm{N}$ stage & 0.933 & $0.351-2.455$ & 0.888 & & & \\
\hline TNM stage & 2.027 & $0.947-4.337$ & 0.069 & & & \\
\hline Radiotherapy response & 18.467 & $7.580-44.993$ & $<0.001$ & 19.368 & $7.641-49.095$ & $<0.001$ \\
\hline
\end{tabular}

PFS, progression-free survival; ALDH1B1, aldehyde dehydrogenase 1B1.

Table 4 Cox regression for OS analysis

\begin{tabular}{|c|c|c|c|c|c|c|}
\hline Characteristics & \multicolumn{3}{|c|}{ Univariate } & \multicolumn{3}{|c|}{ Multivariate } \\
\hline Age (years) & 3.296 & $1.185-9.163$ & 0.022 & 1.151 & $0.503-4.781$ & 0.445 \\
\hline Sex & 2.483 & $0.723-8.528$ & 0.149 & & & \\
\hline Smoking & 0.859 & $0.345-2.138$ & 0.744 & & & \\
\hline T stage & 1.365 & $0.536-3.472$ & 0.514 & & & \\
\hline $\mathrm{N}$ stage & 1.771 & $0.637-4.923$ & 0.273 & & & \\
\hline TNM stage & 2.223 & $0.892-5.541$ & 0.086 & & & \\
\hline Radiotherapy response & 5.300 & $2.072-13.558$ & 0.001 & 4.073 & $1.569-10.570$ & 0.004 \\
\hline
\end{tabular}

OS, overall survival; ALDH1B1, aldehyde dehydrogenase 1B1.

have the potential diagnostic value for NPC patients. The further analyses indicated that ALDH1B1 expression was significantly correlated to age and radiotherapy response. Elderly patients had a significantly high expression of ALDH1B1 compared with younger patients (66.7\% vs. $20.6 \%$, respectively). Consistent with what was expected before, patients with high ALDH1B1 expression are more likely to resistant to radiotherapy. Kaplan-Meier survival analysis revealed that patients with high ALDH1B1 expression had an unfavorable prognosis both in PFS and OS. In addition, ALDH1B1 expression were also the independent prognostic factors for OS in locally advanced NPC patients.

Notably, our research also showed some limitations. Since the study was a retrospective analysis of small sample size, larger sample size is needed to confirm the results. Only locally advanced NPC patients were enrolled in this study, other stage of NPC patients should be considered in future study. Furthermore, the present study did not investigate the function of ALDH1B1 in vivo and in vitro experiments, and the underlying mechanism of ALDH1B1 in tumorigenesis and radiation resistance should be 
performed.

\section{Conclusions}

We have found that ALDH1B1 was correlated with radioresistance in locally advanced NPC patients. The high expression of ALDH1B1 predicted unfavorable survival both in OS and PFS. The results may provide a novel prognostic marker and a potential therapeutic target for locally advanced NPC.

\section{Acknowledgments}

We would like to thank Prof. Xiao-Ting Liu (PalmDrive Inc.) for her help in polishing our paper.

Funding: This study was supported by Hunan Province Natural Science Foundation (No. 2020JJ4543).

\section{Footnote}

Reporting Checklist: The authors have completed the REMARK reporting checklist. Available at https://tcr. amegroups.com/article/view/10.21037/tcr-21-1979/rc

Data Sharing Statement: Available at https://tcr.amegroups. com/article/view/10.21037/tcr-21-1979/dss

Conflicts of Interest: All authors have completed the ICMJE uniform disclosure form (available at https://tcr.amegroups. com/article/view/10.21037/tcr-21-1979/coif). The authors have no conflicts of interest to declare.

Ethical Statement: The authors are accountable for all aspects of the work in ensuring that questions related to the accuracy or integrity of any part of the work are appropriately investigated and resolved. This study was conducted in accordance with the Declaration of Helsinki (as revised in 2013) and was approved by the Ethics Committee of The First Affiliated Hospital of University of South China (No. 2020110512007). The requirement for informed consent was waived.

Open Access Statement: This is an Open Access article distributed in accordance with the Creative Commons Attribution-NonCommercial-NoDerivs 4.0 International License (CC BY-NC-ND 4.0), which permits the noncommercial replication and distribution of the article with the strict proviso that no changes or edits are made and the original work is properly cited (including links to both the formal publication through the relevant DOI and the license). See: https://creativecommons.org/licenses/by-nc-nd/4.0/.

\section{References}

1. Bray F, Ferlay J, Soerjomataram I, et al. Global cancer statistics 2018: GLOBOCAN estimates of incidence and mortality worldwide for 36 cancers in 185 countries. CA Cancer J Clin 2018;68:394-424.

2. Chen YP, Chan ATC, Le QT, et al. Nasopharyngeal carcinoma. Lancet 2019;394:64-80.

3. Hui EP, Ma BBY, Chan ATC. The emerging data on choice of optimal therapy for locally advanced nasopharyngeal carcinoma. Curr Opin Oncol 2020;32:187-95.

4. Johnson D, Ma BBY. Targeting the PD-1/ PD-L1 interaction in nasopharyngeal carcinoma. Oral Oncol 2021;113:105127.

5. Lee AW, Ma BB, Ng WT, et al. Management of Nasopharyngeal Carcinoma: Current Practice and Future Perspective. J Clin Oncol 2015;33:3356-64.

6. Garcia-Mayea Y, Mir C, Masson F, et al. Insights into new mechanisms and models of cancer stem cell multidrug resistance. Semin Cancer Biol 2020;60:166-80.

7. Clarke MF. Clinical and Therapeutic Implications of Cancer Stem Cells. N Engl J Med 2019;380:2237-45.

8. Nassar D, Blanpain C. Cancer Stem Cells: Basic Concepts and Therapeutic Implications. Annu Rev Pathol 2016;11:47-76.

9. Wei D, Peng JJ, Gao H, et al. ALDH1 Expression and the Prognosis of Lung Cancer: A Systematic Review and Meta-Analysis. Heart Lung Circ 2015;24:780-8.

10. Kida K, Ishikawa T, Yamada A, et al. Effect of ALDH1 on prognosis and chemoresistance by breast cancer subtype. Breast Cancer Res Treat 2016;156:261-9.

11. Taylor LA, Abraham RM, Tahirovic E, et al. High ALDH1 expression correlates with better prognosis in tumorigenic malignant melanoma. Mod Pathol 2017;30:634-9.

12. Tsochantaridis I, Roupas A, Voulgaridou GP, et al. Aldehyde Dehydrogenase 1B1 Is Associated with Altered Cell Morphology, Proliferation, Migration and Chemosensitivity in Human Colorectal Adenocarcinoma Cells. Biomedicines 2021;9:44.

13. Singh S, Arcaroli J, Chen Y, et al. ALDH1B1 Is Crucial for Colon Tumorigenesis by Modulating Wnt/ $\beta$-Catenin, Notch and PI3K/Akt Signaling Pathways. PLoS One 2015;10:e121648. 
14. Mameishvili E, Serafimidis I, Iwaszkiewicz S, et al. Aldh1b1 expression defines progenitor cells in the adult pancreas and is required for Kras-induced pancreatic cancer. Proc Natl Acad Sci U S A 2019;116:20679-88.

15. Singh S, Arcaroli JJ, Orlicky DJ, et al. Aldehyde Dehydrogenase $1 \mathrm{~B} 1$ as a Modulator of Pancreatic Adenocarcinoma. Pancreas 2016;45:117-22.

16. You Q, Guo H, Xu D. Distinct prognostic values and potential drug targets of ALDH1 isoenzymes in non-small-cell lung cancer. Drug Des Devel Ther 2015;9:5087-97.

17. Eisenhauer EA, Therasse P, Bogaerts J, et al. New response evaluation criteria in solid tumours: revised RECIST guideline (version 1.1). Eur J Cancer 2009;45:228-47.

18. Wan FZ, Chen KH, Sun YC, et al. Exosomes overexpressing miR-34c inhibit malignant behavior and reverse the radioresistance of nasopharyngeal carcinoma. J Transl Med 2020;18:12.

19. Dai Y, Zhang Y, Yang M, et al. Radiosensitivity-Related Genes and Clinical Characteristics of Nasopharyngeal Carcinoma. Biomed Res Int 2020;2020:1705867.

20. Langan RC, Mullinax JE, Ray S, et al. A Pilot Study Assessing the Potential Role of non-CD133 Colorectal
Cancer Stem Cells as Biomarkers. J Cancer 2012;3:231-40.

21. Tang Z, Li C, Kang B, et al. GEPIA: a web server for cancer and normal gene expression profiling and interactive analyses. Nucleic Acids Res 2017;45:W98-W102.

22. Batlle E, Clevers H. Cancer stem cells revisited. Nat Med 2017;23:1124-34.

23. Yang L, Shi P, Zhao G, et al. Targeting cancer stem cell pathways for cancer therapy. Signal Transduct Target Ther 2020;5:8.

24. da Silva LP, Lopes MLDS, Sarmento ASC, et al. Increased expression of ALDH-1 is associated with clinical parameters of salivary glands neoplasms. Exp Mol Pathol 2020;117:104552.

25. Ginestier C, Hur MH, Charafe-Jauffret E, et al. ALDH1 is a marker of normal and malignant human mammary stem cells and a predictor of poor clinical outcome. Cell Stem Cell 2007;1:555-67.

26. Singh S, Brocker C, Koppaka V, et al. Aldehyde dehydrogenases in cellular responses to oxidative/ electrophilic stress. Free Radic Biol Med 2013;56:89-101.

27. Wang X, Li C, Yao W, et al. MicroRNA-761 suppresses tumor progression in osteosarcoma via negatively regulating ALDH1B1. Life Sci 2020;262:118544.
Cite this article as: Zhu T, He JY, Liu YP, Deng K, Zuo JH, Ai XH. ALDH1B1 predicts poor survival for locally advanced nasopharyngeal carcinoma patients. Transl Cancer Res 2022;11(2):382-391. doi: 10.21037/tcr-21-1979 